\title{
Rectus abdominis muscle injuries in elite handball players: management and rehabilitation
}

This article was published in the following Dove Press journal:

Open Access Journal of Sports Medicine

4 July 2011

Number of times this article has been viewed

\author{
Ramon Balius' \\ Carles Pedret ${ }^{2}$ \\ Laura Pachecol \\ Josep Antoni Gutierrez \\ Joan Vives ${ }^{4}$ \\ Jaume Escoda \\ 'Consell Català de l'Esport, \\ Generalitat de Catalunya, Barcelona; \\ ${ }^{2}$ Centro Mapfre de Medicina del Tenis, \\ Barcelona; ${ }^{3}$ Spanish National Handball \\ Team, Spain; ${ }^{4}$ Granollers Handball \\ Club, Granollers, Barcelona, Spain
}

Correspondence: Ramon Balius Consell Català de l'Esport, Generalitat de Catalunya,

Barcelona, Spain

Tel +34 67862467l

Email ramonbaliusmatas@gmail.com

\begin{abstract}
Muscle injuries generally occur in two-joint muscles with a high percentage of type II fibers during the performance of eccentric activity. Some muscle injuries, such as those located in the adductor longus, a monoarticular muscle, as well as rectus abdominis do not fully comply with these requirements. This study examines five cases of elite handball players with ruptured rectus abdominals. Sonographically, lesions in rectus abdominis are shown as a disruption of the fibrillar pattern with a hematic suffusion that invades the entire lesion. In some of the cases, the ultrasound study was complemented with a MRI. A unified rehabilitation protocol was applied and the return to play time of each handball player ranged between 16 and 22 days, with an average of 18.2 days. Follow-up at 15 months showed no evidence of re-injury or residual discomfort and all of them are playing at their highest level. The aim of this study was to illustrate a feature of handball injury that, as in tennis and volleyball, is uncommon and so far has not been specifically reported. The phenomenon of contralateral abdominal hypertrophy in handball appears in the dominant arm as in tennis and volleyball.
\end{abstract}

Keywords: rectus abdominis, muscle injury, sonography, MRI, handball

\section{Introduction}

The rectus abdominis are paired, strap-like muscles which are separated by a midline band known as the linea alba. Each muscle has two tendinous origins: a medial head which originates from the anterior surface of the pubic symphysis and a larger lateral portion which originates from the upper border of the crest. Together, these constitute the muscle mass which inserts into the fifth, sixth, and seventh costal cartilages. $^{1}$

The muscle is crossed by three transverse fibrous bands or tendinous intersections which blend inseparably with the anterior layer of the rectus sheath. These are located at the level of the umbilicus, the xiphoid process, and midway between the two. ${ }^{1,2}$

Muscle injuries typically occur in muscles which cross two joints with a high percentage of type II fibers during eccentric muscle activity. ${ }^{3}$ Some muscle injuries, such as those occurring in the adductor longus (monoarticular muscle) and the rectus abdominis itself, however, do not fully meet these prerequisites. ${ }^{3}$ Injuries to the rectus abdominis were reported some years ago in the context of batting and throwing sports. ${ }^{3}$ These injuries are thus found in nonarticular and multilaminated muscle, produced by an eccentric contraction mechanism typical of highly specific sports movements such as service, smash, or spike in tennis or volleyball. ${ }^{3}$ 
The aim of this study is to illustrate an aspect of handballrelated injury which, as is the case with tennis and volleyball, is uncommon and has not been specifically reported to date. This feature is exemplified with five cases of elite handball players who suffered a ruptured rectus abdominis.

\section{Materials and methods}

During the period 2005 to 2008, five cases of acute myotendinous injury to the rectus abdominis were diagnosed using a Toshiba Xario (SSA660) musculoskeletal ultrasound machine (Toshiba Medical Systems, Tokyo, Japan) at the Consell Català de l'Esport Barcelona (Spain) and underwent treatment with the medical services provided by their club. The five cases studied were elite handball players from the Spanish league and all players were participants in European competitions. All of the handball players involved in this study signed an informed consent form and granted permission for their data to be published.

In addition, 17 asymptomatic elite handball players from the Spanish national handball team (mean age 24.76 years; height $190.82 \mathrm{~cm}$; weight $91.18 \mathrm{~kg}$ ) were recruited to undergo a sonographic examination of rectus abdominis thickness. The rectus thickness was thus measured in each player, the abdominal wall musculature evaluated, and a comparison of rectus abdominis thickness made on either side by measuring the distance between the posterior and anterior margins at maximum thickness level below the umbilical line.

\section{Cases}

\section{Case I: Professional handball player, 28 years old, left-handed}

During a match, this player created an eccentric contraction of the rectus abdominis while making a throwing gesture with the ipsilateral arm. The player suddenly took a shot between two opponents who were blocking his waist, having overcome the block with his upper body to throw the ball ipsilaterally. Ultrasonography was performed 36 hours later and an injury of $26 \mathrm{~mm}$ (long axis) and $12 \mathrm{~mm}$ (short axis) observed. The injury was situated in the deep epimysium, $28 \mathrm{~mm}$ below the umbilical line. Twenty-four hours later, an MRI was performed which showed a focal high signal consistent with the location indicated by the ultrasound. Following rehabilitation therapy, the player resumed sports activities within 17 days.

\section{Case 2: Elite junior handball player, 16 years old, left-handed}

During a match, the player experienced a sudden pain as a result of the eccentric contraction of the rectus abdominis muscle while performing a throwing motion with the contralateral arm. An ultrasound was performed 72 hours later, which showed a full thickness injury of about $35 \mathrm{~mm}$ (long axis) and $20 \mathrm{~mm}$ (short axis), located $10 \mathrm{~mm}$ below the umbilical line. Following rehabilitation therapy, the patient resumed sports activities within 20 days.

\section{Case 3: Professional handball player, 27 years old, right-handed}

During a match, the player experienced a sudden pain as the result of an eccentric contraction of the rectus abdominis muscle while performing a throwing motion with the contralateral arm. The natural throwing gesture was abruptly blocked by an opponent. An ultrasound was performed 36 hours later which showed an injury of about $35 \mathrm{~mm}$ (long axis) and $20 \mathrm{~mm}$ (short axis). The injury was located in the deep epimysium, $24 \mathrm{~mm}$ below the umbilical line. Thirty-six hours later, an MRI was performed which showed a focal high signal in a location consistent with the findings obtained by ultrasound. Following rehabilitation therapy, the player was able to resume sports activities within 16 days.

\section{Case 4: Professional handball player, 28 years old, left-handed}

During training, the player experienced a sudden pain due to an eccentric contraction of the rectus abdominis muscle as he performed a throwing motion with the contralateral arm. An ultrasound was performed 48 hours later which showed an injury of about $16 \mathrm{~mm}$ (long axis) and $10 \mathrm{~mm}$ (short axis), located in the deep epimysium $19 \mathrm{~mm}$ below the umbilical line. Following rehabilitation therapy, the player was able to resume sports activities within 16 days.

\section{Case 5: Professional handball player, 21 years old, left-handed}

During a match, the player experienced a sudden pain due to an eccentric contraction of the rectus abdominis muscle as he performed a throwing motion with the contralateral arm. The natural throwing gesture was abruptly blocked by an opponent. An ultrasound was performed 48 hours later which showed a full thickness injury of about $17 \mathrm{~mm}$ (long axis) and $19 \mathrm{~mm}$ (short axis), located $14 \mathrm{~mm}$ below the umbilical line. Following rehabilitation therapy, the player was able to resume sports activities within 22 days.

\section{Sonographic features}

In all cases, the sonographic assessment of the injury in the rectus abdominis consistently presented as a fibrillar 
disruption, hematic suffusion invading the entire lesion. In addition to the fibrillar defect, edema and hemorrhage were likewise identified (Figure 1).

\section{Rehabilitation protocol}

Although there is no universally accepted functional rehabilitation program for this kind of injury according to the existing literature, ${ }^{4}$ the two clubs in which the rehabilitation program was carried out followed mutually consistent protocols under the supervision of the same physician of the Consell Català de l'Esport (Barcelona, Spain).

In the initial phase, also known as the inflammatory phase (from injury time to 72 hours), patients were ordered to refrain from performing any sports activity and abdominal muscle activation and were given painkiller medication and an ice pack. Active work was initiated on day 4 which involved transverse muscle activation, followed by rectus abdominis and abdominal oblique muscle work in both the isometric and internal directions, consistently paying attention to the rule of discomfort - although not the pain threshold - and subsequently progressing to full-range amplitude movements and eccentric muscle contraction. After a week, a three-step rehabilitation program was established, the phases of which ranged from jogging to throwing. This was followed by physical therapy which focused on affected soft tissue areas. By day 5, patients proceeded to the gradual stretching of the rectus abdominis and oblique muscles in order to prevent the occurrence of pain due to the formation of scar tissue (gradually progressing from passive stretching to active tension). Before moving from one phase to the next, athletes were required to be free of pain. The pace of progress of active work was set according to levels of discomfort experienced rather than pain threshold. In order to ensure appropriate progress, the level of discomfort experienced by patients was qualified as bearable and ranged between $5 \%$ and $10 \%$ of the visual analog scale (Figure 2).

\section{Statistical analysis}

In order to proceed to a general assessment of the differences in the thickness of the different parts of the rectus

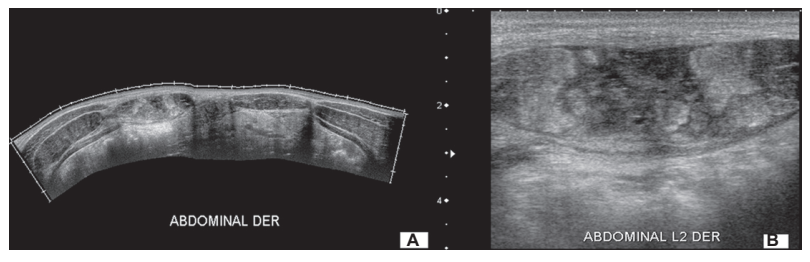

Figure I 16-year-old elite male handball player (case 2). Ultrasound made 4 days after the lesion. (A) Panoramic view of the injury; (B) Short axis cut shows focal area of myofibril disruption of full thickness lamina of the rectus abdominis, with hyperechoic edema.

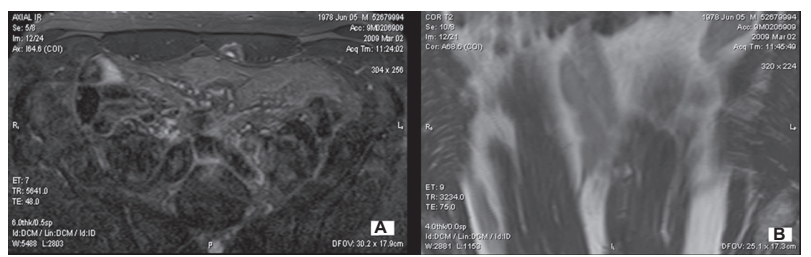

Figure 2 32-year-old male. (A) Axial T2 WI fat suppression (TR/TE, 564I/48); (B) coronal T2 WI (3234/75) images show posterior aspect of left rectus abdominis with focal area of hyperintensity (arrow) compatible with muscle edema, hemorrhage, and fibril disruption (partial tear).

abdominis, a two-factor analysis of variance with repeated measurements (depth and dominance) was performed. Levels were deemed significant when $\alpha=0.05$. A Student $t$-test was used to analyze the differences between the dominant and nondominant hands and the Curran-Everett recommendations used to establish the degree of significance. ${ }^{5}$

\section{Discussion}

There is a current lack of published research devoted to injuries produced during handball activity. Dirx et al presented a casecontrol study on the background of handball injuries among players aged 12 years or older. These injuries were frequently located in the lower extremities (54\%), especially the ankle. The majority of injuries involved distortion (35\%) and strain (26\%), but none were found to involve the abdominal muscles. ${ }^{6}$

Seil et al conducted a prospective study which involved 16 handball teams over the course of a season, the aim of which was to study the incidence of injuries in relation to exposure during matches and practice. The study found 91 injuries, with an incidence of 2.5 injuries per 1000 player-hours; $37 \%$ of the injuries involved the upper extremities and 54\% the lower extremities. The knee was the most commonly injured joint, followed by finger, ankle, and shoulder joints. No injury to the rectus abdominis muscle was reported. ${ }^{7}$

Wedderkopp et al presented a prospective study of 22 women's teams from Denmark, the objective of which was to implement an intervention program designed to reduce the number of injuries in young female players in European handball, with a special emphasis on injuries to the lower extremities. There was no reference to any injury to the rectus abdominis. ${ }^{8}$

Langevoort et al analyzed the injuries suffered by handball players during major international tournaments. This was therefore the first study to be conducted with elite players. In a total of six tournaments, 478 lesions were found, the average incidence being 108 injuries/1000 hours. The injuries most frequently encountered affected the lower extremities (42\%), followed by injuries to the head (23\%), upper extremities $(18 \%)$, and trunk (14\%). ${ }^{9}$ 
The lack of references found in the specialized literature on rectus abdominis injuries in handball contrasts with the knowledge some coaches and senior veteran players have on the subject. Moreover, as Langevoort et al reported, ${ }^{9}$ several authors have analyzed the incidence of handball injuries in amateur players, yet information on handball injuries sustained by top-level players is very limited. As is the case in tennis and volleyball, lesions of this kind appear to be typical of elite players, not amateurs.

Rectus abdominis injuries are typically sustained during the practice of throwing or batting sports and at a level which implies the potential for injury. Surprisingly, despite handball being typically associated with sports such as tennis and volleyball, it is currently poorly referenced in international literature. A very interesting article by Johnson ${ }^{10}$ reported that, anecdotally, abdominal wall injuries are reported as common, yet epidemiological studies fail to support this contention. This is particularly true for volleyball, ${ }^{11}$ where the majority of epidemiologic articles do not identify abdominal injury. ${ }^{12-14}$ One article was found which discussed injuries sustained by professional beach volleyball players and reported the injury as requiring medical attention even though no withdrawal from sports activities was needed..$^{15}$

Several epidemiological and clinical articles have been published on rectus abdominis injury in tennis players. ${ }^{16-18}$ Eccentric overload, followed by the forced contraction of the nondominant rectus abdominis during the cocking phase of the service motion is the accepted injury mechanism. ${ }^{19}$ In this position, injury is consistently sustained in the contralateral rectus abdominis of the armed wing, usually at infraumbilical level. ${ }^{2,19}$

As was the case with tennis, ${ }^{20}$ in our study, the rupture occurred in the arm contralateral to the shooting arm in all cases except one (case 1), in which the rupture occurred in the left rectus abdominis as a shot was taken with the same-side arm. The special circumstances of the game forced the player to shoot in a highly lateralized position. In this position, the ipsilateral rectus abdominis was stretched and an eccentric contraction of the ipsilateral abdominal muscles created in the releasing arm.

In handball, all cases involved the hyperextension of the lumbar spine and stretching of the abdominal muscles which were contracted during the cocking phase of the arm throwing the ball. Spinal flexion subsequently occurred with a powerful contraction of all abdominal muscles. The gesture consistently twisted the spine towards the side presenting the lesion. In contrast with other sports such as tennis and volleyball, ${ }^{11,12,19}$ handball involves direct contact with the opponent, which may in some cases entail modified injury biomechanics. In cases 3 and 5, the players were thus abruptly blocked by an opponent, and in case 1, the player took a sudden shot between two opponents who were blocking his waist, forcing him to correct the direction of the shot, which in turn led to injury to the shooting arm on the ipsilateral side.

A laterality phenomenon which is typically linked with tennis involves a marked hypertrophy of the abdominal muscle on the side contralateral to the dominant arm compared with the ipsilateral muscle. ${ }^{2}$ Connell et al ${ }^{2}$ found significant differences in the anteroposterior diameter of the dominant side $(21.2 \pm 1.93 \mathrm{~mm}[\mathrm{SD}])$ compared with the nondominant side $(28.2 \pm 1.93 \mathrm{~mm})$. The same abdominal hypertrophy phenomenon was observed in handball players. ${ }^{21}$ In 2009, we were able to use ultrasound to examine 17 handball players from the Spanish national team. Significant differences were found in the anteroposterior diameter of the rectus abdominis on either side (Table 1), with the anteroposterior diameter measured on the side of the dominant hand averaging $19.6 \pm 2.94 \mathrm{~mm}$ and $20.9 \pm 3.13 \mathrm{~mm}$ on the side of the nondominant hand. These measurements were markedly lower than those provided by Connell et al in their group of tennis players and showed the diameter measured on the dominant side to be greater than that of the nondominant side. The difference was statistically significant $(P=0.002)$.

The time it took for each handball player to resume playing handball ranged from 16 to 22 days, with a mean time

Table I Anteroposterior diameter comparison of dominant (D) and nondominant (ND) rectus abdominis in elite handball players

\begin{tabular}{llllll}
\hline $\begin{array}{l}\text { Patient } \\
\text { number }\end{array}$ & $\begin{array}{l}\text { Age } \\
(\mathbf{y})\end{array}$ & $\begin{array}{l}\text { Height } \\
(\mathbf{c m})\end{array}$ & $\begin{array}{l}\text { Weight } \\
(\mathbf{k g})\end{array}$ & $\begin{array}{l}\mathbf{D} \\
(\mathbf{m m})\end{array}$ & $\begin{array}{l}\text { ND } \\
(\mathbf{m m})\end{array}$ \\
\hline 1 & 25 & 194 & 98 & 22.3 & 23.2 \\
2 & 24 & 195 & 90 & 17.5 & 18.4 \\
3 & 23 & 176 & 75 & 16.7 & 18.6 \\
4 & 21 & 201 & 107 & 20.7 & 21.8 \\
5 & 31 & 178 & 85 & 22.5 & 23.9 \\
6 & 23 & 184 & 88 & 19.6 & 21.1 \\
7 & 22 & 197 & 107 & 14 & 14.3 \\
8 & 33 & 192 & 90 & 19.9 & 18.2 \\
9 & 26 & 186 & 76 & 18.2 & 19.9 \\
10 & 32 & 194 & 91 & 18.9 & 22.7 \\
11 & 22 & 195 & 110 & 19.3 & 18.9 \\
12 & 21 & 196 & 88 & 22.4 & 23.9 \\
13 & 24 & 194 & 88 & 26.8 & 27 \\
14 & 22 & 199 & 105 & 21.5 & 25 \\
15 & 24 & 187 & 81 & 17.1 & 20.9 \\
16 & 23 & 186 & 84 & 17.8 & 18.2 \\
17 & 25 & 190 & 87 & 18.2 & 20.3 \\
Mean & 24.8 & 190.8 & 91.2 & 19.6 & $\mathbf{2 1 . 0}$ \\
Standard & & & & $\mathbf{8 . 7}$ & $\mathbf{9 . 8}$ \\
deviation & & & & & \\
Pearson's $\boldsymbol{r}$ & & & & $\mathbf{0 . 8 9}$ & \\
& & & & $\mathbf{P = 0 . 0 0 2}$ & \\
\hline
\end{tabular}


span of 18.2 days, a period which was shorter than the 4 to 6 weeks reported by Connell et $\mathrm{al}^{2}$ and the 4 to 5 (mean 2.8) weeks reported by Maquirriain et al. ${ }^{19}$ This may be due to the fact that the number of goal shots typically taken when playing handball is much lower than the number of services typically involved in a tennis match. In addition, the positions required to take a handball shot are both different and more wide-ranging than those observed in the practice of tennis. Although gradual, the players' return to normal activities was very fast. All were able to tolerate the discomfort reported during the first few days of training with the team and none were forced to delay resumption of activities. Follow-up checks carried out at 15 months showed no evidence of re-injury or residual discomfort.

Lastly, indirect mechanism muscle injuries appear to be myoconnective in kind. ${ }^{22}$ In tennis players displaying rectus abdominis muscle rupture, the lesion nearly always affects the connective posterior plane in the deep epimysium. ${ }^{21}$ This fact was also noted in all of the cases involved in our study.

\section{Conclusion}

There is a lack of published research on injuries sustained during handball practice. The aim of our study was to illustrate a lesion which, although rare, is typical of high-level handball, tennis, and volleyball, and has not been specifically reported in handball to date. This study examined five cases of ruptured rectus abdominis muscles in handball players. The tear occurred in the contralateral shooting arm in 4 of the cases and all of the injuries where located at infraumbilical level.

In handball, the phenomenon of contralateral abdominal hypertrophy of the dominant arm was also observed.

\section{Acknowledgment}

Dr Galilea Piero (High Performance Sports Centre. Sant Cugat del Vallès. Barcelona) for performing the statistical analysis.

\section{Disclosure}

The authors report no conflicts of interest in this work.

Open Access Journal of Sports Medicine

\section{Publish your work in this journal}

Open Access Journal of Sports Medicine is an international, peer-reviewed, open access journal publishing original research, reports, reviews and commentaries on all areas of sports medicine. The manuscript management system is completely online and includes a very quick and fair peer-review system.

\section{References}

1. Sinnatamby CS, editor. Lat's Anatomy, 10th ed. Edinburgh, Scotland: Churchill Livingtone; 1999:218.

2. Connell D, Ali K, Javid M, et al. Sonography and MRI of rectus abdominis muscle strain in elite tennis players. AJR Am J Roentgenol. 2006; 187:1457-1461.

3. Garrett WE Jr. Muscle strain injuries. Am J Sports Med. 1996; 24 (Suppl 6):2-8.

4. Cross TM, Gibbs N, Houang M, et al. Acute quadriceps muscle strains: magnetic resonance imaging features and prognosis. Am J Sports Med. 2004;32;710-719.

5. Curran-Everett D. Multiple comparisons: philosophies and illustrations. Am J Physiol Regulatory Integrative Comp Physiol. 2000; 279:R1-R8.

6. Dirx M, Bouter LM, de Geus GH. Aetiology of handball injuries: a case - control study. Br J Sports Med. 1992;26;121-124.

7. Seil R, Rupp S, Tempelhof S, et al. Sports injuries in team handball. A one-year prospective study of sixteen men's senior teams of a superior nonprofessional level. Am J Sports Med. 1998;26:681-687.

8. Wedderkopp N, Kaltoft M, Lundgaard B, et al. Prevention of injuries in young female players in European team handball. A prospective intervention study. Scand J Med Sci Sports. 1999;9:41-47.

9. Langevoort G, Myklebust G, Dvorak J, et al. Handball injuries during major international tournaments. Scand J Med Sci Sports. 2007; 17:400-407.

10. Johnson R. Abdominal wall injuries: rectus abdominis strains, oblique strains, rectus sheath hematoma. Current Sports Medicine Reports. 2006;5:99-103.

11. Aagaard H, Jorgensen U. Injuries in elite volleyball. Scand J Med Sci Sports. 1996;6:228-232

12. Bahr R, Bahr A. Incidence of acute volleyball injuries: a prospective cohort study of injury mechanisms and risk factors. Scand J Med Sci Sports. 1997;7:166-171.

13. Zetou E, Malliou P, Lola A, et al. Factors related to the incidence of injuries appearance to volleyball players. J Back Musculoskelet Rehabil. 2006; 19:129-134.

14. Augustsson SR, Augustsson J, Thomeé R, et al. Injuries and preventive actions in elite Swedish volleyball. Scand J Med Sci Sports. 2006; 6:433-440.

15. Bahr R, Reeser JC. Injuries among world-class professional beach volleyball players. Am J Sports Med. 2003;31:119-125.

16. Balduini FC. Abdominal and groin injuries in tennis. Clin Sports Med. 1988;7:5-9.

17. Lehman RC. Thoracoabdominal musculoskeletal injuries in racquet sports. Clin Sports Med. 1988;7:267-276.

18. Kibler W, Chandler J. Racquet sports. In: Fu FH, Stone DA, editors. Sports Injuries - Mechanism, Prevention, and Treatment. Baltimore, MD: Williams \& Wilkins; 1994:278-292.

19. Maquirriain J, Ghisi JP, Kokalj AM. Rectus abdominis muscle strains in tennis players. Br J Sports Med. 2007;41;842-848.

20. Bylak J, Hutchinson MR. Common sports injuries in young tennis players. Sports Med. 1998;26:119-132.

21. Sanchis-Moysi J, Idoate F, Dorado C, et al. Large asymmetric hypertrophy of rectus abdominis muscle in professional tennis players. PLoS One. 2010;5(12):e15858. Doi:10.1371/journal.pone.0015858.

22. Medical services Futbol Club Barcelona. Clinical Practice Guide for muscular injuries. Epidemiology, diagnosis, treatment and prevention. Apunts Med Esport. 2009;164:179-203.

\section{Dovepress}

Visit http://www.dovepress.com/testimonials.php to read real quotes from published authors. 